\title{
Congenitally missing second permanent molars in non-syndromic patients (Review)
}

\author{
ALEXANDRU-TITUS FARCAȘIU ${ }^{1 *}$, RODICA LUCA ${ }^{2}$, ANDREEA DIDILESCU $^{3 *}$, IOANA-ANDREEA STANCIU $^{2}$, \\ CATALINA FARCASIU ${ }^{2}$, ARINA VINEREANU ${ }^{2 *}$ and ANETA MUNTEANU ${ }^{2}$
}

Departments of ${ }^{1}$ Removable Prosthodontics, ${ }^{2}$ Pedodontics and ${ }^{3}$ Embryology, Faculty of Dental Medicine,

'Carol Davila' University of Medicine and Pharmacy, Bucharest 032799, Romania

Received September 15, 2021; Accepted October 15, 2021

DOI: $10.3892 / \mathrm{etm} .2021 .11068$

\begin{abstract}
Hypodontia (tooth agenesis) is regarded as the most common congenital dental anomaly. The present review discusses the epidemiological characteristics of congenitally missing second permanent molars (CMSPMs) within a systematic review of the literature. The review was based on Pubmed library associated with the search of various scientific databases or academic resources, improved by hand search of reference lists. The terms 'hypodontia' or 'anodontia' in combination with 'prevalence' or 'epidemiology' were searched in the data sources for studies published between January 2001 and December 2020. Abstracts of non-English papers were also analyzed. The inclusion criteria were as follows: i) Study provided precise data about CMSPMs, even if no second permanent molar was reportedly missing; ii) the number of CMSPMs distributed by jaw was provided and iii) studies on subjects $>3$ years were used. The exclusion criteria were as follows: i) Studies on patients with history of trauma of the maxilla or the mandible, any type of syndrome affecting bone metabolism, metabolic disorders, previous extraction or tooth loss due to dental caries, cleft lip and palate; ii) studies performed on cohorts of patients with hypodontia and iii) studies reporting data including third molars, except for those that presented sufficient data to perform correct calculations. A total of 79 studies were selected, accumulating a population of 281,968 people, with an average sample size of 3,524.60 $\pm 11,255.25$. The prevalence of CMSPMs (IpHSPM) was $2.79 \pm 3.16 \%$ among all missing teeth $(1.03 \pm 1.59 \%$ for upper CMSPMs and
\end{abstract}

Correspondence to: Dr Alexandru-Titus Farcașiu, Department of Removable Prosthodontics, Faculty of Dental Medicine, 'Carol Davila' University of Medicine and Pharmacy, Aleea Barajul Iezer nr. 8, Bucharest 032799, Romania

E-mail: alexandru.farcasiu@umfed.ro

\section{*Contributed equally}

Key words: hypodontia, second permanent molar, prevalence, non-syndromic patients, epidemiology
$1.76 \pm 2.32 \%$ for lower CMSPMs; $\mathrm{P}=0.011)$. There were no significant differences $(\mathrm{P}=0.250)$ in IpHSPM between men $(1.59 \pm 1.52 \%)$ and women $(2.13 \pm 1.67 \%)$. However, significant differences were recorded between continents. Furthermore, lower CMSPMs were found more frequently in orthodontic samples $(\mathrm{P}=0.033)$. The prevalence of CMSPMs is low compared with the overall prevalence of CM teeth. Despite the rarity of these anomalies, early detection is important to enable practitioners to plan and start treatment at the best time for optimal results.

\section{Contents}

1. Introduction

2. Data selection

3. Studies reporting data on CMSPMs

4. Epidemiological characteristics of CMSPMs

5. Conclusions

\section{Introduction}

Oral health plays a major role in public health. Congenitally missing (CM) teeth, one of the most frequent dental anomalies (1-3), negatively affects both aesthetics and function, and may require a combination of expensive orthodontic, prosthodontic and surgical treatments (4-6). Hypodontia of permanent teeth is associated with several complications, such as malocclusion with mastication problems, periodontal trauma, reduced growth of the alveolar bone, difficult pronunciation and unfavorable aesthetics (7-9). Hypodontia is determined by disturbances of the early stages of tooth development (10). Local pathology or trauma, endocrine disorders or developmental anomalies are also considered etiological factors (11). A polygenic mode of inheritance is the most supported etiological theory, involving epistatic genes and environmental factors influencing phenotypic expression $(10,12)$. Dental agenesis may be caused by mutations in several genes, including MSX, PAX9, TGFA and AXIN2 (13-17). Recently, the WNT10A gene was reported as a major candidate gene for non-syndromic hypodontia (18). Genes involved in tooth agenesis are associated with 
colorectal cancer $(19,20)$ and ovarian cancer $(21)$; however, the exact mechanisms remain unclear (22). Understanding the molecular mechanism may help identify an efficient protocol for the early detection of cancer in patients with hypodontia.

In 2004, Mattheeuws et al (23) suggested that hypodontia was diagnosed more often during the 20th century. Larmour et al (24) reported a maximum hypodontia prevalence of $11.3 \%$ with racial differences regarding the most affected tooth. Notably, almost all studies reported higher incidence of hypodontia in women (23-25). Khalaf et al (25) reported that the worldwide overall prevalence of hypodontia in permanent teeth was $6.4 \%$, with statistically significant differences between continents. Prevalence of hypodontia in Africa was the highest (13.4\%), followed by Europe (7\%), Asia (6.3\%) and Australia (6.3\%), while North America (5\%) and Latin America and Caribbean (4.4\%) exhibited the lowest values (25).

Hypodontia in permanent dentition can be an isolated anomaly (26) or associated with various genetic syndromes affecting the head and neck region (27). In non-syndromic patients, the absence of second permanent molars (SPM) is rare, similar to that of canines and first molars, and mostly occurs in mandibular arch (25). According to the American Association of Pediatric Dentistry, the calcification of SPM begins at 30-36 months, with the completion of enamel mineralization at 7-8 years (28). Thus, radiological studies regarding the congenital absence of SPM should be performed after 3 years (28).

Review data regarding the CMSPMs among all missing teeth is scarce. The present review discusses the epidemiological characteristics of CMSPMs within a systematic review of the literature.

\section{Data selection}

For the present review, the search was based on Pubmed library associated with the search of various scientific databases (SpingerLink, https://link.springer.com/; Nature, https://www.nature.com/; Wiley Online, https://onlinelibrary. wiley.com/; and ScienceDirect, https://www.sciencedirect. $\mathrm{com} /$ ) or academic resources (ResearchGate, Academia and SematicScholar). Hand search of existing reference lists and Google search consistently improved the depth of the present review. The terms 'hypodontia' or 'anodontia' and 'prevalence' or 'epidemiology' were searched in the data sources for studies since 2001. Abstracts of non-English papers were also analyzed. The literature review was performed in February 2021. PRISMA guidelines (29) were followed when performing this review.

The inclusion criteria were as follows: i) Precise data about CMSPMs was mandatory for inclusion even if no SPM was missing; ii) the number of missing SPMs distributed by location was required; iii) sex distribution of missing SPM was registered if available; iv) only radiological studies on cohorts of patients $>3$ years were used and v) only studies published since 2001 were eligible. The exclusion criteria were as follows: i) All studies had to exclude patients with a history of trauma of the maxilla or the mandible, any type of syndrome affecting bone metabolism, metabolic disorders, previous extraction or tooth loss due to dental caries, cleft lip and palate; ii) studies on cohorts of patients with hypodontia were excluded and iii) studies only based on clinical evaluation were excluded.

The data collected data included authors, year of publication, area/city of study, country and continent of study, characteristics of the study population, number of participants, mean age or age limits, sex distribution, prevalence of overall hypodontia, number of CMSPMs and prevalence of CMSPMs by jaw and by sex (when it was available).

A total of 79 studies were selected. All data presented within selected articles were checked for calculating errors, and double digits were used throughout the review. When data were available, the prevalence of CMSPM was calculated for men and women, respectively. CMSPMs values were calculated as percentages of all CM teeth. Given that CMSPMs are rare, corresponding authors were contacted if published data suggested the existence of non-mentioned missing molar teeth. Statistical analyses were performed using ANOVA, independent samples t-test, and correlation analyses were performed using SPSS 19.0 software (IBM Corp.). $\mathrm{P}<0.05$ was considered to indicate a statistically significant difference. The review was submitted for registration at PROSPERO (no. 281147).

\section{Studies reporting data on CMSPMs}

Studies reporting no CMSPMs $(n=30)(2,30-58)$ were performed on cohorts that ranged from 250-6,015 patients (mean value, 1,723.55 $\pm 1,421.56$ ), presenting a prevalence of hypodontia (IpH) of $6.94 \%$ (Table I).

Studies with CMSPMs $(\mathrm{n}=49)(3,33,48,59-104)$ were performed on cohorts that ranged from 139-100,577 patients, with a mean sample size of $4,627.70 \pm 14,133.27$. The mean value of prevalence of IpH was $8.52 \pm 10.13 \%$ for 228,916 evaluated patients. Among all $\mathrm{CM}$ teeth, the prevalence of CMSPMs (IpHSPM) was $4.47 \pm 2.91 \%$. A total of 25/50 studies provided data regarding sex distribution of CMSPMs, while IpH was calculated for 47 studies (two studies reported a hypodontia prevalence including third molars) (Table II).

\section{Epidemiological characteristics of CMSPMs}

Studies reporting no CMSPMs. For this category of studies, the highest mean value of IpH was found in Asia (7.74\%), followed by Europe (5.88\%), South America (4.79\%) and Africa (4.68\%). Most of the studies were based on Asian populations $(n=19)$, six were performed in Europe, three in Africa and one in South America. Studies on orthodontic patients were most frequent $(n=12 ; 40 \%)$, followed by studies on pediatric dentistry patients $(n=4)$, sampled studies $(n=4)$, general practice patients $(n=3)$, orthodontic and pediatric dentistry patients $(n=3)$, radiological patients $(n=2)$ and non-orthodontic patients/medical students $(\mathrm{n}=1)$. Regarding the type of study group, radiological patients had the highest prevalence of IpH (16.45\%), followed by sampled studies (7.51\%), orthodontic patients $(6.62 \%)$, pediatric patients (6.58\%), orthodontic and pediatric dentistry patients $(6.31 \%)$, non-orthodontic patients $(5.50 \%)$, general practice patients 
Table I. Studies reporting no CMSPMs.

\begin{tabular}{|c|c|c|c|c|c|c|}
\hline First author/s, year & Location & $\begin{array}{c}\text { Study } \\
\text { population }\end{array}$ & $\begin{array}{l}\text { No. } \\
\text { patients }\end{array}$ & $\begin{array}{l}\text { Age range/mean } \\
\text { age, years }\end{array}$ & $\mathrm{IpH}$ & (Refs.) \\
\hline $\begin{array}{l}\text { Ng'ang'a and Ng'ang'a, } \\
2001\end{array}$ & Nairobi/Kenya & OP & 615 & $8.00-15.00$ & 6.30 & (30) \\
\hline $\begin{array}{l}\text { Albashaireh and Khader, } \\
2006\end{array}$ & Irbid/Jordan & NOP & 1,005 & $14.00-46.00$ & 5.50 & (31) \\
\hline $\begin{array}{l}\text { Altug-Atac and Erdem, } \\
2007\end{array}$ & Ankara/Turkey & OP & 3,043 & $8.50-14.75$ & 2.62 & (2) \\
\hline Küchler et al, 2008 & Rio de Janeiro/Brazil & PP & 1,167 & $6.00-12.00 / 8.90$ & 4.79 & (32) \\
\hline Harris and Clark, 2008 & Memphis/United States & $\mathrm{OP}$ & 600 & $12.00-18.00$ & 36.29 & (33) \\
\hline Aslam et al, 2010 & Rawalpindi/Pakistan & OP & 1,185 & $\begin{array}{c}12.00-37.00 / \\
18.11\end{array}$ & 4.30 & (34) \\
\hline Ajami et al, 2010 & Mashhad/Iran & SQ & 600 & $9.00-14.00 / 10.63$ & 9.00 & (35) \\
\hline Bud et al, 2011 & Târgu-Mures/Romania & $\mathrm{OP}$ & 804 & $11.00-21.00$ & 6.84 & (36) \\
\hline Amini et al, 2012 & Teheran/Iran & OP & 3,374 & $\begin{array}{l}10.00-20.00 / \\
13.90\end{array}$ & 5.21 & (4) \\
\hline Sheikhi et al, 2012 & Multiple regions/Iran & SQ & 2,422 & $7.00-35.00 / 9.30$ & 10.90 & $(37)$ \\
\hline Cantekin et al, 2012 & Erzurum/Turkey & $\mathrm{OP} / \mathrm{PP}$ & 1,291 & $8.00-14.00$ & 6.19 & (38) \\
\hline $\begin{array}{l}\text { Al-Jabaa and Aldrees, } \\
2013\end{array}$ & Riyadh/Saudi Arabia & OP & 602 & $\begin{array}{l}\text { Permanent } \\
\text { dentition }\end{array}$ & 19.10 & (39) \\
\hline Mani et al, 2014 & Kuala Lumpur/Malaisya & $\mathrm{RP}$ & 834 & $12.00-16.00$ & 27.21 & $(40)$ \\
\hline $\begin{array}{l}\text { Affasn and Serrour, } \\
2014\end{array}$ & Khartoum/Sudan & MS & 2,401 & $\begin{array}{l}\text { Permanent } \\
\text { dentition }\end{array}$ & 2.66 & $(41)$ \\
\hline Hassan et al, 2014 & Khartoum/Sudan & OP & 1,069 & $>8.00$ & 5.10 & $(42)$ \\
\hline Kramerova et al, 2014 & Olomouc/Czech Rep & $\mathrm{PP}$ & 434 & $8.00-19.00$ & 9.45 & $(43)$ \\
\hline Shokri et al, 2014 & Hamadan/Iran & $\mathrm{RP}$ & 1,649 & $7.00-35.00 / 21.79$ & 5.70 & (44) \\
\hline Majeed et al, 2014 & Karachi/Pakistan & OP & 250 & $\begin{array}{c}12.00-37.00 / \\
18.11\end{array}$ & 2.85 & $(45)$ \\
\hline Al Jawad et al, 2015 & Doha/Qatar & $\mathrm{OP} / \mathrm{PP}$ & 1,269 & $8.00-20.00 / 11.60$ & 6.22 & $(46)$ \\
\hline Zhang et al, 2015 & Hebei/China & GP & 6,015 & $10.00-26.00$ & 5.89 & $(47)$ \\
\hline $\begin{array}{l}\text { Hashim and Al-Said, } \\
2016\end{array}$ & Doha/Qatar & OP & 1,000 & $\begin{array}{l}10.00-26.00 / \\
16.40\end{array}$ & 7.80 & $(48)$ \\
\hline Gokkaya and Kargul, 2016 & Istanbul/Turkey & PP & 1,658 & $7.00-12.00$ & 6.15 & (49) \\
\hline Sajjad et al, 2016 & Al-Jouf/Saudi Arabia & OP & 1,267 & $9.00-30.00 / 16.77$ & 6.10 & $(50)$ \\
\hline Al-Abdallah et al, 2015 & Amman/Jordan & $\mathrm{OP} / \mathrm{PP}$ & 3,315 & $8.60-25.40 / 17.40$ & 6.54 & $(51)$ \\
\hline Ameen et al, 2017 & Erbil/Iraq & OP & 600 & $10.00-34.00$ & 6.66 & $(52)$ \\
\hline Sola et al, 2018 & Madrid/Spain & $\mathrm{RS}$ & 2,500 & $8.00-11.00$ & 3.48 & $(53)$ \\
\hline Reshitaj et al, 2019 & Kosovo (8 regions) & $\mathrm{RS}$ & 3,306 & $15.00-21.00$ & 6.66 & (54) \\
\hline Georgescu et al, 2019 & Bucharest/Romania & GP & 755 & $<18.00 / 10.20$ & 2.91 & $(55)$ \\
\hline Musaed et al, 2019 & Multiple regions/Yemen & GP & 5,100 & $9.00-25.00 / 15.00$ & 3.23 & $(56)$ \\
\hline Farcaşiu et al, 2020 & Bucharest/Romania & PP & 453 & 8.75 & 5.96 & $(57)$ \\
\hline
\end{tabular}

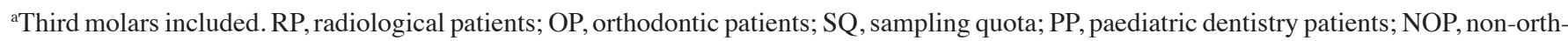
odontic patients; SC, school children; GP, general practice; MS, medical students; IpH, prevalence of hypodontia.

(4.01\%) and medical students $(2.66 \%)$. No significant correlations were observed between the prevalence of hypodontia and type of study $(\mathrm{P}=0.097)$, sample size $(\mathrm{P}=0.245)$ or continent $(\mathrm{P}=0.061)$. 
Table II. Prevalence of agenesis of SPMs in non-syndromic patients: Distribution by arch and sex in studies reporting at least one CMSPM.

\begin{tabular}{|c|c|c|c|c|c|c|c|c|}
\hline $\begin{array}{l}\text { First author/s, } \\
\text { year }\end{array}$ & Location & $\begin{array}{c}\text { Study } \\
\text { population }\end{array}$ & $\begin{array}{c}\text { No. } \\
\text { patients }\end{array}$ & $\mathrm{IpH}$ & $\begin{array}{c}\text { Age } \\
\text { range/mean } \\
\text { age, } \\
\text { years }\end{array}$ & $\begin{array}{c}\text { Upper } \\
\text { IpHSPMs } \\
(\%) \\
\text { M/F (n) }\end{array}$ & $\begin{array}{c}\text { Lower } \\
\text { IpHSPMs } \\
(\%) \\
\text { M/F (n) }\end{array}$ & (Refs.) \\
\hline Fekonja, & Maribor/Slovenia & $\mathrm{OP}$ & 212 & 11.32 & 12.70 & 0.00 & $\begin{array}{c}2.08 \\
0 / 1\end{array}$ & (58) \\
\hline $\begin{array}{l}\text { Endo et al, } \\
2006\end{array}$ & Niigata/Japan & OP & 3,358 & 8.51 & $\begin{array}{l}5.00- \\
15.00\end{array}$ & $\begin{array}{l}3.01 \\
8 / 13\end{array}$ & $\begin{array}{c}4.31 \\
13 / 17\end{array}$ & (59) \\
\hline $\begin{array}{l}\text { Sisman et al, } \\
2007\end{array}$ & $\begin{array}{l}\text { Kayseri\& } \\
\text { Kirrikale/Turkey }\end{array}$ & OP & 2,413 & 7.54 & $9.00-36.00$ & $\begin{array}{c}1.32 \\
1 / 4\end{array}$ & $\begin{array}{l}1.32 \\
3 / 2\end{array}$ & $(60)$ \\
\hline $\begin{array}{l}\text { Maatouk et al, } \\
2008\end{array}$ & Sayada/Tunisia & PP & 262 & 13.30 & $\begin{array}{c}12.00- \\
18.00 / 14.10\end{array}$ & 1.61 & 1.61 & (61) \\
\hline $\begin{array}{l}\text { Goya et al, } \\
2008\end{array}$ & Matsudo/Japan & OP & 2,072 & 9.74 & $\begin{array}{c}3.00- \\
17.00 / 9.40\end{array}$ & $\begin{array}{l}4.35 \\
6 / 19\end{array}$ & $\begin{array}{l}1.91 \\
2 / 9\end{array}$ & (3) \\
\hline $\begin{array}{l}\text { Abu Sakra } \\
\text { and Alqaqaa, } \\
2008\end{array}$ & Amman/Jordan & $\mathrm{OP}+\mathrm{PP}$ & 1,524 & 4.40 & $8.00-20.00$ & 1.01 & 1.01 & (62) \\
\hline $\begin{array}{l}\text { Chung et al, } \\
2008\end{array}$ & Seoul/Korea & OP & 1,622 & 11.20 & $>10.00$ & 0.60 & 1.20 & (8) \\
\hline $\begin{array}{l}\text { Harris and } \\
\text { Clark 2008a }\end{array}$ & Memphis/USA & OP & 1,100 & 36.29 & $12.00-18.00$ & $\begin{array}{l}1.81 \\
2 / 1\end{array}$ & $\begin{array}{c}2.42 \\
4 / 0\end{array}$ & (33) \\
\hline $\begin{array}{l}\text { Rølling and } \\
\text { Poulsen, } 2009\end{array}$ & Aarhus/Denmark & $\mathrm{SC}$ & 8,138 & 7.38 & $9.00-12.00$ & $\begin{array}{l}1.21 \\
2 / 11\end{array}$ & $\begin{array}{l}2.05 \\
9 / 13\end{array}$ & (63) \\
\hline $\begin{array}{l}\text { Aktan et al, } \\
2010\end{array}$ & 6 regions/Turkey & GP & 100,577 & 3.12 & $5.00-37.00$ & $\begin{array}{c}0.85 \\
12 / 15\end{array}$ & $\begin{array}{c}1.01 \\
13 / 19\end{array}$ & (9) \\
\hline $\begin{array}{l}\text { Pekker et al, } \\
2009\end{array}$ & Ankara/Turkey & $\mathrm{RP}$ & 139 & 73.38 & $10.00-71.00$ & $\begin{array}{c}5.07 \\
6 / 7\end{array}$ & $\begin{array}{c}3.51 \\
5 / 4\end{array}$ & (64) \\
\hline $\begin{array}{l}\text { Gomes et al, } \\
2010\end{array}$ & Brasilia/Brazil & OP & 1,049 & 6.29 & $\begin{array}{c}10.00- \\
15.70 / 13.16\end{array}$ & $\begin{array}{c}0.92 \\
0 / 1\end{array}$ & $\begin{array}{c}3.70 \\
1 / 3\end{array}$ & (65) \\
\hline $\begin{array}{l}\text { Topkara and } \\
\text { Sari, } 2011\end{array}$ & Selcuk/Turkey & OP & 2,761 & 6.77 & $\begin{array}{c}9.00- \\
46.00 / 14.10\end{array}$ & $\begin{array}{c}3.20 \\
4 / 8\end{array}$ & $\begin{array}{l}1.60 \\
3 / 3\end{array}$ & $(66)$ \\
\hline $\begin{array}{l}\text { Vahid-Dasterji } \\
\text { et al, } \\
2010\end{array}$ & Teheran/Iran & OP & 1,751 & 9.13 & $\begin{array}{c}9.00- \\
27.00 / 12.50\end{array}$ & 1.36 & 2.72 & $(67)$ \\
\hline $\begin{array}{l}\text { Tallón } \\
\text {-Walton et al, } \\
2010\end{array}$ & Girona/Spain & GP & 1,518 & 7.25 & $6.00-83.00$ & 4.71 & 4.71 & (68) \\
\hline $\begin{array}{l}\text { Carvalho et al, } \\
2011\end{array}$ & Porto/Portugal & OP & 139 & 6.47 & $8.00-17.00$ & 0.00 & $\begin{array}{l}6.25 \\
0 / 1\end{array}$ & (69) \\
\hline Kim, 2011 & Seoul/Korea & $\mathrm{OP}$ & 3,055 & 11.30 & $9.00-30.00$ & 3.00 & 0.76 & (70) \\
\hline $\begin{array}{l}\text { Kazanci et al, } \\
2011\end{array}$ & Erzurum/Turkey & OP & 3,165 & 4.29 & $\begin{array}{c}9.00- \\
25.00 / 14.17\end{array}$ & 0.00 & 1.30 & (71) \\
\hline $\begin{array}{l}\text { Masamichi } \\
\text { et al, } 2011\end{array}$ & Kanto/Japan & PP & 2,125 & 11.80 & $7.00-20.00$ & 4.26 & 2.22 & $(72)$ \\
\hline $\begin{array}{l}\text { Coelho et al, } \\
2012\end{array}$ & Porto/Portugal & PP & 1,438 & 7.99 & $\begin{array}{c}6.00- \\
15.00 / 8.82\end{array}$ & 1.87 & 1.12 & (73) \\
\hline Medina, 2012 & Carcas/Venezuela & OP & 607 & 4.11 & $5.00-11.00$ & 0.00 & 8.00 & (74) \\
\hline $\begin{array}{l}\text { de Freitas et al, } \\
2012 \mathrm{a}\end{array}$ & Sao Paolo/Brazil & OP & 512 & 9.18 & $6.00-20.00$ & 8.47 & 3.38 & $(75)$ \\
\hline $\begin{array}{l}\text { Uzuner et al, } \\
2013\end{array}$ & Ankara/Turkey & OP & 2,530 & 4.98 & $7.00-16.00$ & $\begin{array}{c}0.39 \\
1 / 0\end{array}$ & $\begin{array}{l}1.17 \\
2 / 1\end{array}$ & $(76)$ \\
\hline $\begin{array}{l}\text { González- } \\
\text { Allo et al, } 2012\end{array}$ & Portugal & GP & 2,888 & 6.05 & $\begin{array}{c}7.00- \\
21.00 / 14.06\end{array}$ & $\begin{array}{c}2.34 \\
7 / 0\end{array}$ & $\begin{array}{l}6.04 \\
8 / 10\end{array}$ & (77) \\
\hline
\end{tabular}


Table II. Continued.

\begin{tabular}{|c|c|c|c|c|c|c|c|c|}
\hline $\begin{array}{l}\text { First author/s, } \\
\text { year }\end{array}$ & Location & $\begin{array}{c}\text { Study } \\
\text { population }\end{array}$ & $\begin{array}{c}\text { No. } \\
\text { patients }\end{array}$ & IpH & $\begin{array}{c}\text { Age } \\
\text { range/mean } \\
\text { age, } \\
\text { years }\end{array}$ & $\begin{array}{c}\text { Upper } \\
\text { IpHSPMs } \\
(\%) \\
\text { M/F (n) }\end{array}$ & $\begin{array}{c}\text { Lower } \\
\text { IpHSPMs } \\
(\%) \\
\text { M/F (n) }\end{array}$ & (Refs.) \\
\hline $\begin{array}{l}\text { Hyunsoon } \\
\text { et al, } 2013\end{array}$ & Cheonbuk/Korea & PP & 3,302 & 5.39 & $7.00-15.00$ & $\begin{array}{l}3.23 \\
13 / 5\end{array}$ & $\begin{array}{l}2.87 \\
13 / 3\end{array}$ & (78) \\
\hline $\begin{array}{l}\text { Kerekes-Máthé } \\
\text { et al, } 2013\end{array}$ & $\begin{array}{l}\text { Târgu-Mureș } \\
\text { Romania }\end{array}$ & GP & 947 & 7.39 & $\begin{array}{c}9.00- \\
34.00 / 16.51\end{array}$ & 0.00 & 0.73 & (79) \\
\hline $\begin{array}{l}\text { Al-Amiri et al, } \\
2013\end{array}$ & New York/USA & OP & 496 & 9.47 & 16.30 & 1.96 & 1.96 & (80) \\
\hline $\begin{array}{l}\text { Hedayati and } \\
\text { Dashlibrun, } \\
2013\end{array}$ & Shiraz/Iran & $\mathrm{OP}$ & 494 & 7.66 & $10.00-18.00$ & $\begin{array}{l}1.07 \\
1 / 0\end{array}$ & $\begin{array}{l}4.30 \\
2 / 2\end{array}$ & (81) \\
\hline $\begin{array}{l}\text { Bozga et al, } \\
2014\end{array}$ & Bucharest/Romania & OP & 518 & 6.75 & $6.00-41.00$ & 0.00 & 6.38 & (82) \\
\hline $\begin{array}{l}\text { Herrera- } \\
\text { Atoche et al, } \\
2014\end{array}$ & Yucatan/Mexico & OP & 670 & 5.82 & $9.00-20.00$ & 0.00 & 10.60 & (83) \\
\hline $\begin{array}{l}\text { Pop Acev } \\
\text { and Gjorgova, } \\
2014\end{array}$ & $\begin{array}{l}\text { FYROM } \\
\text { regions/FYROM }\end{array}$ & OP & 8,160 & 7.52 & $\begin{array}{c}8.00- \\
18.00 / 11.29\end{array}$ & $\begin{array}{l}1.03 \\
2 / 11\end{array}$ & $\begin{array}{l}2.95 \\
9 / 28\end{array}$ & (84) \\
\hline $\begin{array}{l}\text { Zhang et al, } \\
2015\end{array}$ & Beijing/China & OP & 2,781 & 7.48 & $10.00-26.00$ & $\begin{array}{c}2.38 \\
9 / 2\end{array}$ & $\begin{array}{l}1.51 \\
5 / 2\end{array}$ & (47) \\
\hline $\begin{array}{l}\text { Abu-Hussein } \\
\text { et al, } 2015\end{array}$ & Almothalath/Israel & GP & 2,200 & 2.59 & $\begin{array}{c}12.00- \\
39.50 / 16.20\end{array}$ & 0.59 & 1.19 & (85) \\
\hline $\begin{array}{l}\text { Dang et al, } \\
2017\end{array}$ & $\begin{array}{l}\text { New South } \\
\text { Wales/Australia }\end{array}$ & $\mathrm{SC}$ & 1,050 & 5.14 & $\begin{array}{c}6.00- \\
18.00 / 10.20\end{array}$ & 0.00 & 8.69 & (86) \\
\hline $\begin{array}{l}\text { Hagiwara et al, } \\
2016\end{array}$ & Tokyo/Japan & $\mathrm{SC}$ & 9,584 & 3.88 & $16.00-18.00$ & $\begin{array}{c}0.64 \\
2 / 2\end{array}$ & 0.00 & (87) \\
\hline $\begin{array}{l}\text { Badrov et al, } \\
2017\end{array}$ & Split reg./Croatia & OP & 4,430 & 7.78 & $\begin{array}{c}6.00- \\
15.00 / 10.60\end{array}$ & $\begin{array}{l}1.33 \\
3 / 6\end{array}$ & $\begin{array}{c}5.05 \\
13 / 21\end{array}$ & (88) \\
\hline $\begin{array}{l}\text { Gracco et al, } \\
2017\end{array}$ & Padua region/Italy & $\mathrm{OP}$ & 4,006 & 8.96 & $9.00-16.00$ & $\begin{array}{l}1.42 \\
5 / 5\end{array}$ & $\begin{array}{c}2.99 \\
11 / 10\end{array}$ & (89) \\
\hline $\begin{array}{l}\text { Laganà et al, } \\
2017\end{array}$ & Rome/Italy & NOP & 4,706 & 7.11 & $\begin{array}{c}8.00- \\
12.00 / 9.60\end{array}$ & $\begin{array}{l}1.91 \\
6 / 6\end{array}$ & $\begin{array}{c}2.95 \\
12 / 11\end{array}$ & (90) \\
\hline $\begin{array}{l}\text { Souza- } \\
\text { Silva et al, } \\
2018\end{array}$ & Sergipe/Brazil & $\mathrm{OP}$ & 2,239 & 3.03 & $8.00-30.00$ & 1.75 & 2.63 & (91) \\
\hline $\begin{array}{l}\text { Ifesanya et al, } \\
2018\end{array}$ & Ibadan/Nigeria & $\mathrm{OP}$ & 216 & 10.18 & $\begin{array}{c}10.00- \\
46.00 / 14.00\end{array}$ & 5.55 & 1.81 & (92) \\
\hline $\begin{array}{l}\text { Altan et al, } \\
2019\end{array}$ & Antakya/Turkey & PP & 9,831 & 2.80 & $8.00-14.00$ & 0.00 & $\begin{array}{c}0.96 \\
0 / 6\end{array}$ & (93) \\
\hline & Gaziosmanpasa/Turkey & PP & 11,372 & 1.63 & & 0.00 & $\begin{array}{l}1.01 \\
2 / 2\end{array}$ & \\
\hline $\begin{array}{l}\text { Fauzi et al, } \\
2019\end{array}$ & $\begin{array}{l}\text { Pahang Darul } \\
\text { Makmur/Malayzia }\end{array}$ & GP & 3,481 & 1.00 & $\begin{array}{c}12.00- \\
60.00 / 23.53\end{array}$ & $\begin{array}{l}1.33 \\
0 / 1\end{array}$ & 0.00 & (94) \\
\hline $\begin{array}{l}\text { Allhadad et al, } \\
2019\end{array}$ & Kerbala/Iraq & GP & 1,150 & $30.60^{\mathrm{a}}$ & $\begin{array}{c}9.00- \\
44.00 / 21.20\end{array}$ & $\begin{array}{c}0.69 \\
4 / 0\end{array}$ & 0.00 & (95) \\
\hline $\begin{array}{l}\text { Kielan- } \\
\text { Grabowska et al, } \\
2019\end{array}$ & Wroclaw/Poland & $\mathrm{OP}$ & 674 & 11.57 & $6.00-15.00$ & 2.94 & 7.64 & (96) \\
\hline $\begin{array}{l}\text { Wiener and } \\
\text { Waters, } 2019\end{array}$ & $\begin{array}{l}\text { West Virginia/ } \\
\text { USA }\end{array}$ & PP & 500 & 12.00 & $6.00-12.00$ & 1.48 & 3.70 & (97) \\
\hline
\end{tabular}


Table II. Continued.

\begin{tabular}{|c|c|c|c|c|c|c|c|c|}
\hline $\begin{array}{l}\text { First author/s, } \\
\text { year }\end{array}$ & Location & $\begin{array}{c}\text { Study } \\
\text { population }\end{array}$ & $\begin{array}{c}\text { No. } \\
\text { patients }\end{array}$ & $\mathrm{IpH}$ & $\begin{array}{c}\text { Age } \\
\text { range/mean } \\
\text { age, } \\
\text { years }\end{array}$ & $\begin{array}{c}\text { Upper } \\
\text { IpHSPMs } \\
(\%) \\
\text { M/F (n) }\end{array}$ & $\begin{array}{c}\text { Lower } \\
\text { IpHSPMs } \\
(\%) \\
\text { M/F (n) }\end{array}$ & (Refs.) \\
\hline $\begin{array}{l}\text { Wiener and } \\
\text { Waters, } 2019\end{array}$ & $\begin{array}{l}\text { West Virginia/ } \\
\text { USA }\end{array}$ & PP & 500 & 12.00 & $6.00-12.00$ & 1.48 & 3.70 & (97) \\
\hline $\begin{array}{l}\text { Chan et al, } \\
2019\end{array}$ & Singapore & $\mathrm{OP}$ & 2,508 & 11.10 & $14.00-25.00$ & 1.68 & 1.12 & (98) \\
\hline $\begin{array}{l}\text { De Stefani et al, } \\
2019\end{array}$ & Padua/Italy & $\mathrm{OP}$ & 600 & 9.00 & 9.00 & $\begin{array}{c}1.00 \\
-48.00\end{array}$ & 3.00 & (99) \\
\hline $\begin{array}{l}\text { Gupta and } \\
\text { Rauniyar, } 2020\end{array}$ & Kathmandu/Nepal & OP & 601 & 7.48 & $\begin{array}{c}10.00 \\
-35.00 / 16.42\end{array}$ & 0.00 & 2.77 & (100) \\
\hline $\begin{array}{l}\text { Çakir and } \\
\text { Yildirim, } 2020\end{array}$ & Selcuk/Turkey & PP & 9,950 & 1.51 & $5.00-14.00$ & $\begin{array}{c}0.30 \\
0 / 1\end{array}$ & $\begin{array}{c}0.60 \\
0 / 2\end{array}$ & (101) \\
\hline
\end{tabular}

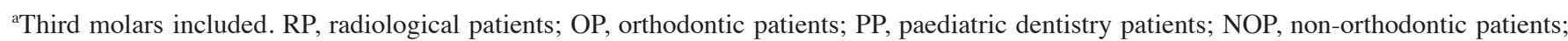
SC, school children; GP, general practice; IpH, prevalence of hypodontia; SPMs, second permanent molars.

Table III. Hypodontia and CMSPMs in selected studies (\%).

\begin{tabular}{lccccccc}
\hline Variable & Worldwide & North America & Asia & Africa & Europe & Australia & South America \\
\hline IpH & $7.91 \pm 8.54$ & $10.73 \pm 1.78$ & $8.50 \pm 11.15$ & $7.50 \pm 4.22$ & $7.39 \pm 2.10$ & 5.14 & $4.80 \pm 1.31$ \\
IpHSPMs & $2.79 \pm 3.16$ & $2.50 \pm 2.40$ & $1.85 \pm 2.36$ & $2.11 \pm 3.24$ & $3.67 \pm 3.39$ & 8.69 & $6.57 \pm 4.42$ \\
Upper CMSPMs & $1.03 \pm 1.59$ & $0.98 \pm 0.89$ & $0.93 \pm 1.39$ & $1.43 \pm 2.40$ & $0.98 \pm 1.28$ & 0.00 & $1.85 \pm 3.31$ \\
Lower CMSPMs & $1.76 \pm 2.32$ & $1.57 \pm 1.63$ & $0.91 \pm 1.20$ & $0.64 \pm 0.93$ & $2.68 \pm 2.53$ & 8.69 & $4.71 \pm 3.86$ \\
Males CMSPMs & $1.59 \pm 1.52$ & 0.97 & $1.64 \pm 1.55$ & - & $1.65 \pm 1.76$ & - & 0.92 \\
Females CMSPMs & $2.21 \pm 1.65$ & 0.15 & $1.77 \pm 1.52$ & - & $3.30 \pm 1.48$ & - & 3.70 \\
\hline
\end{tabular}

IpH, prevalence of hypodontia; CMSPMs, congenitally missing second permanent molars; -, not available.

Studies with CMSPMs. Hypodontia was most prevalent in Africa (11.74\%), followed by North America (10.73\%), Asia (9.11\%), Europe (8.03\%), Australia (5.14\%) and South America (4.81\%). Most of the studies were based on Asian populations ( $n=24), 14$ were performed in Europe, five in South America, three in North America, two in Africa and one in Australia. Most studies were performed on orthodontic samples $(n=29 ; 58 \%)$, followed by pediatric dentistry patients $(n=9 ; 16 \%)$, general practice patients $(n=7 ; 14 \%)$, school children $(n=3 ; 6 \%)$, radiological patients $(n=2$; $4 \%)$ and non-orthodontic patients $(\mathrm{n}=1 ; 2 \%)$. IpH was highest in radiological studies $(38.89 \%)$, followed by orthodontic samples (7.96\%), non-orthodontic patients $(7.11 \%)$, pediatric dentistry patients $(7.05 \%)$, school children $(5.46 \%)$ and general practice patients $(4.56 \%)$. Hypodontia values were significantly associated with the type of study group $(\mathrm{P}<0.001)$. Statistical analysis demonstrated that radiological patients presented a significantly higher prevalence of hypodontia $(\mathrm{P}<0.001)$ compared with orthodontic patients, who had more missing teeth than general practice samples $(\mathrm{P}=0.004)$.

No statistically significant differences were observed between studies reporting or not missing SPMs regarding the prevalence of hypodontia ( 8.52 vs. $6.94 \%$; $\mathrm{P}=0.369)$ or mean number of subjects within the respective categories of studies $(1,686.10$ vs. $4,627.70 ; \mathrm{P}=0.151)$, although studies reporting CMSPMs had greater values for $\mathrm{IpH}$.

All selected studies cumulated a population of 281,968 people, with an average sample size of $3,524.60 \pm 11,255.25$. The highest prevalence of hypodontia was found in North America, followed by Asia, Africa, Europe, Australia and South America. CMSPMs had an overall prevalence of $2.79 \pm 3.16 \%$ among all missing teeth $(1.03 \pm 1.59 \%$ for upper SPMs and $1.76 \pm 2.32 \%$ for lower SPMs; $\mathrm{P}=0.011)$. Only 25 studies reported sex values. There were no significant differences $(\mathrm{P}=0.250)$ between men $(1.59 \pm 1.52 \%)$ and women $(2.13 \pm 1.67 \%)$. European studies presented significantly higher differences for mean values of lower vs. upper CMSPMs ( $\mathrm{P}=0.009)$. Lower SPM was missing more frequently in Europe compared with Asia $(\mathrm{P}<0.001)$ and Africa $(\mathrm{P}=0.010)$, and in South America compared with Asia $(\mathrm{P}<0.001)$. Furthermore, European women had more CMSPMs compared with Northern American women $(\mathrm{P}=0.016)$ (Table III).

The prevalence of $\mathrm{IpH}$ was highest for radiological patients, with a significant ANOVA test $(\mathrm{P}=0.001)$ vs. all other types of cohorts, which were statistically similar. However, 
Table IV. Hypodontia and CMSPMs by study type (\%).

\begin{tabular}{|c|c|c|c|c|c|c|c|}
\hline Variable & $\mathrm{RP}$ & $\mathrm{OP}$ & SQ & PP & $\mathrm{OP}+\mathrm{PP}$ & NOP & $\mathrm{SC}$ \\
\hline $\mathrm{IpH}$ & $27.65 \pm 32.21$ & $7.58 \pm 3.10$ & $7.51 \pm 3.19$ & $6.89 \pm 4.05$ & $6.31 \pm 0.19$ & $6.30 \pm 1.13$ & $5.46 \pm 1.77$ \\
\hline IpHSPM & $2.65 \pm 4.06$ & $3.51 \pm 3.27$ & 0.00 & $2.23 \pm 2.48$ & 0.00 & $2.43 \pm 3.43$ & $4.19 \pm 4.10$ \\
\hline
\end{tabular}

RP, radiological patients; OP, orthodontic patients; SQ, sampling quota; PP, paediatric dentistry patients; NOP, non-orthodontic patients; $\mathrm{SC}$, school children; IpH, prevalence of hypodontia; IpHSPM, prevalence of congenitally missing second permanent molar.

Table V. Comparison of hypodontia and CMSPMs between orthodontic samples and all other type of cohorts (\%).

\begin{tabular}{lcccrrr}
\hline Type of cohort & IpH & $\begin{array}{c}\text { Upper } \\
\text { CMSPM }\end{array}$ & $\begin{array}{c}\text { Lower } \\
\text { CMSPM }\end{array}$ & $\begin{array}{c}\text { Males } \\
\text { CMSPM }\end{array}$ & $\begin{array}{c}\text { Females } \\
\text { CMPSM }\end{array}$ \\
\hline Orthodontic & $7.58 \pm 3.10$ & $1.20 \pm 1.78$ & $2.31 \pm 2.54$ & $0.54 \pm 0.97$ & $0.94 \pm 1.65$ \\
Other & $8.40 \pm 11.88$ & $0.89 \pm 1.39$ & $1.21 \pm 1.95$ & $0.46 \pm 1.28$ & $0.44 \pm 0.97$ & $2.10 \pm 2.93$
\end{tabular}

IpH, prevalence of hypodontia; CMSPMs, congenitally missing second permanent molars.

there were no significant differences regarding the prevalence of CMSPMs depending on the population type (ANOVA; $\mathrm{P}=0.311$ ) (Table IV). Orthodontic cohorts had a lower IpH prevalence than the mean value for other cohorts. CMSPMs were more frequently observed in orthodontic samples, the difference being significant for lower CMSPMs $(\mathrm{P}=0.033)$ and overall $(\mathrm{P}=0.045)$ (Table V).

European orthodontic samples had significantly higher prevalence values for lower CMSPM $(\mathrm{P}=0.029)$ and for $\mathrm{IpH}$ $(\mathrm{P}=0.035)$ compared with other samples, in agreement with Rakhshan (102). European samples' prevalence values in orthodontic cohorts for lower CMSPMSs were significantly higher than Asian $(\mathrm{P}=0.009)$ and African $(\mathrm{P}=0.009)$ values. Overall missing CMSPMs $(\mathrm{P}=0.017)$ were significantly higher in Europe compared with Asia. IpH in orthodontic cohorts was significantly higher in Europe compared with South America $(\mathrm{P}=0.006)$. Northern Americans (Caucasians) in cohorts other than the orthodontic ones had significantly lower CMSPMs compared with Asians $(\mathrm{P}=0.006)$ (Table VI).

Most European studies exhibited more missing lower SPMs with the exception of two studies from Iberian countries. Tallón-Walton et al (68) reported a similar involvement of upper and lower SPMs, while Coelho et al (73) reported more missing upper SPMs. Analysis demonstrated that European women had more CMSPMs than men, except for two studies, González-Allo et al (77) and Gracco et al (89). For European studies, only the mean values from Italy exhibited more missing SPMs in men. All studies reported a higher prevalence of lower CMSPMs, except for Spain (Table VII).

A total of two studies from Italy $(68,90)$, performed in the same year (2017) in Rome (non-orthodontic patients) and the Padua region (orthodontic patients) reported similar values for CMSPMs. Sexes were affected similarly regarding upper CMSPM, while there were more lower CMSPMs in men. A total of two Romanian studies reporting CMSPMs were performed in different regions of the country. Both studies reported lower CMSPMs, with different prevalence, 6.83\% (79) and 0.73\% (82).

The situation varied for Asian studies. Data from Iran, Israel and Nepal, and mean values for Turkey sustained a higher prevalence for lower CMSPMs, whilst reports from Iraq, Japan, Korea and Malaysia supported higher prevalence of upper CMSPMs. A certain pattern was set by Korea and Japan, with more upper CMSPMs compared with Asia and Turkey (Table VIII).

There were seven studies form various areas of Turkey, two of which reported more CMSPM in the maxilla compared with the mandible $(64,66)$, although they were based in different regions (Ankara-central Turkey vs. Selcuk, Eastern Mediterranean Coast). The other five studies reported a higher proportion of CMSPMs in the lower arch. A large cohort study performed by Altan et al (93) in two distant regions of Turkey confirmed regional differences regarding sex and maxillary/mandibular distribution of CMSPMs.

Studies from Korea and China reported similar values (3.88 and $3.89 \%$, respectively), while research from Japan exhibited the highest continental prevalence of CMSPMs (5.17\%). Notably, studies from Korea and China reported more missing SPMs in men, while studies from Japan reported more women with missing SPMs. South American studies found a 2.5 times higher absence of lower SPMs compared with upper SPMs. Only one of the five studies had sex-related results, with women 4 times more affected by CMSPMs than men. There are few reviews regarding $\mathrm{IpH}$ in recent years. Mattheeuws et al (23) reviewed data published since 1936 on Caucasian patients, and confirmed that hypodontia has been diagnosed more often in recent studies. Only studies with $>1,000$ examined children were selected. The prevalence of hypodontia ranged between $0.1-10.1 \%$ and was slightly higher for girls.

A significant increase was noted in 1956, which may be due to improvements in imaging and increasing dental awareness or, according to Brook (103), due to a yet 
Table VI. Comparison of IpH and IpHSPM between orthodontic samples and all other types of cohorts by continent (\%).

\begin{tabular}{|c|c|c|c|c|c|c|c|}
\hline Continent & Sample, $\mathrm{n}$ & CMSPMs & $\begin{array}{c}\text { Upper } \\
\text { CMSMP }\end{array}$ & $\begin{array}{c}\text { Lower } \\
\text { CMSPM }\end{array}$ & $\begin{array}{c}\text { Males } \\
\text { CMSPM }\end{array}$ & $\begin{array}{l}\text { Females } \\
\text { CMSPM }\end{array}$ & $\mathrm{IpH}$ \\
\hline \multirow[t]{2}{*}{ Europe } & Orthodontic, 9 & $4.87 \pm 2.99$ & $0.85 \pm 0.99$ & $4.02 \pm 2.44$ & $0.61 \pm 1.01$ & $1.95 \pm 2.21$ & $8.46 \pm 1.91$ \\
\hline & Other, 11 & $2.69 \pm 3.50$ & $1.09 \pm 1.51$ & $1.60 \pm 2.12$ & $0.55 \pm 1.51$ & $0.50 \pm 1.15$ & $6.51 \pm 1.89$ \\
\hline \multirow[t]{2}{*}{ Asia } & Orthodontic, 21 & $2.30 \pm 2.33$ & $1.06 \pm 1.36$ & $1.23 \pm 1.35$ & $0.70 \pm 1.14$ & $0.81 \pm 1.49$ & $7.70 \pm 3.63$ \\
\hline & Other, 23 & $1.44 \pm 2.36$ & $0.81 \pm 1.43$ & $0.62 \pm 1.00$ & $0.50 \pm 1.30$ & $0.49 \pm 1.96$ & $8.87 \pm 15.09$ \\
\hline \multirow[t]{2}{*}{ Africa } & Orthodontic, 3 & $2.45 \pm 4.24$ & $1.85 \pm 3.20$ & $0.60 \pm 1.04$ & - & - & $7.19 \pm 2.65$ \\
\hline & Other, 1 & 3.22 & 1.61 & 1.61 & - & - & 13.30 \\
\hline \multirow[t]{2}{*}{ North America } & Orthodontic, 3 & $1.60 \pm 2.01$ & $0.81 \pm 1.02$ & $0.86 \pm 0.99$ & $0.32 \pm 0.56$ & $0.10 \pm 0.86$ & $3.15 \pm 5.46$ \\
\hline & Other, 1 & 12.00 & 1.48 & 3.70 & - & - & 12.00 \\
\hline \multirow[t]{2}{*}{ South America } & Orthodontic, 5 & $7.89 \pm 3.39$ & $2.22 \pm 3.56$ & $5.66 \pm 3.46$ & $0.18 \pm 0.41$ & $0.74 \pm 1.65$ & $3.85 \pm 2.51$ \\
\hline & Other, 1 & 0.00 & - & - & - & - & 4.79 \\
\hline \multirow[t]{2}{*}{ Australia } & Orthodontic, 0 & - & - & - & - & - & - \\
\hline & Other, 1 & 8.69 & 0.00 & 8.69 & - & - & 5.14 \\
\hline
\end{tabular}

IpH, prevalence of hypodontia; CMSPMs, congenitally missing second permanent molars; -, not available.

Table VII. CMSPMs data in European studies (\%).

\begin{tabular}{|c|c|c|c|c|c|}
\hline Country & IpHSPMs & $\begin{array}{c}\text { Upper } \\
\text { CMSPMs }\end{array}$ & $\begin{array}{c}\text { Lower } \\
\text { CMPSMs }\end{array}$ & $\begin{array}{c}\text { Males } \\
\text { CMSPMs }\end{array}$ & $\begin{array}{c}\text { Females } \\
\text { CMPSMs }\end{array}$ \\
\hline Croatia & 6.24 & 1.33 & 4.91 & 2.38 & 4.01 \\
\hline Denmark & 3.26 & 1.21 & 2.05 & 1.02 & 2.24 \\
\hline Italy & 4.42 & 1.43 & 2.98 & 2.27 & 2.13 \\
\hline N. Macedonia & 3.97 & 1.03 & 2.94 & 0.87 & 3.10 \\
\hline Poland & 10.58 & 2.94 & 7.64 & - & - \\
\hline Portugal & 5.87 & 1.40 & 4.47 & 2.51 & 4.80 \\
\hline Romania & 3.55 & 0.00 & 3.55 & - & - \\
\hline Slovenia & 2.08 & 0.00 & 2.08 & 0.00 & 2.08 \\
\hline Spain & 9.42 & 4.71 & 4.71 & - & - \\
\hline
\end{tabular}

IpHSPMs, prevalence of congenitally missing second permanent molars; CMSPMs, congenitally missing second permanent molars; -, not available.

unidentified environmental factor influencing the phenotype. Larmour et al (24) reported a prevalence of hypodontia between 2.6-11.3\%. The authors reported race-depending variations regarding the most common missing tooth with Caucasians more frequently missing the mandibular second premolars and maxillary lateral incisors, while Asians were more frequently missing the mandibular incisors, and a prevalence ratio of $3 / 2$ in favor of women (24). However, neither of these reviews made any reference to CMSPMs.

In a meta-analysis regarding the prevalence of agenesis of permanent teeth in Caucasian populations, Polder et al (104) reported a higher prevalence of hypodontia for both sexes in Europe (men, 4.6\% and women, 6.3\%) and Australia (men, 5.5\% and women, 7.6\%) compared with Caucasians in North American (men, 3.2\% and women, 4.6\%). For a total of 112,334 people, the authors found 11,422 missing teeth, of which 208 were SPMs (67 upper CMSPMs, $0.58 \%$ and 141 lower CMSPMs, 1.23\%) (104).
Bondemark and Tsiopa reported a prevalence of $0.8 \%$ CMSPMs in a sample of 1543 Swedish children aged 10-16 years. A total of 23 CMSPMs (15 lower and 8 upper SPMs) were missing in 12 children, with a lower/upper ratio of 1.87 (105). Khalaf et al (25) performed a systematic review and meta-analysis on studies published between 2002-2012. Similarly, the authors analyzed the abstracts of non-English papers. The prevalence of hypodontia varied significantly by continent, unlike data reported by Rakhshan and Rakhshan (106), and was the highest in Africa, followed by Europe. Women were found to have a higher prevalence than men. A total of 39/93 studies were taken from two previous systematic reviews $(23,104)$. Khalaf et al $(25)$ reported a prevalence of $1.8 \%$ for lower CMSPMs while upper CMSPMs represented $1.5 \%$ of the total number of missing teeth.

The current study has the highest mean value of sample size and the greatest value of $\mathrm{IpH}$, confirming the trend of 
Table VIII. Prevalence of CMSPMs in Asia (\%).

\begin{tabular}{lcccc}
\hline Country & IpHSPMs & $\begin{array}{c}\text { Upper } \\
\text { CMSPMs }\end{array}$ & $\begin{array}{c}\text { Lower } \\
\text { CMSPMs }\end{array}$ & $\begin{array}{c}\text { Males } \\
\text { CMSPMs }\end{array}$ \\
\hline Japan & 5.17 & 3.05 & 2.12 & 1.57 \\
CMSPMs
\end{tabular}

Not all studies reported sex data. IpHSPMs, prevalence of congenitally missing second permanent molars; CMSPMs, congenitally missing second permanent molars; -, not available.

Table IX. Current research data comparison with previous reviews.

\begin{tabular}{lccccc}
\hline Author, year & $\begin{array}{c}\text { Mean sample } \\
\text { size }\end{array}$ & $\begin{array}{c}\text { Number of studies } \\
\text { taken into account }\end{array}$ & IpH & IpHSPM & $\begin{array}{c}\text { Lower/Upper } \\
\text { CMSPMs ratio }\end{array}$ \\
\hline Polder et al, 2004 & 3404.00 & 33 & 4.83 & 1.82 & 2.00 \\
Khalaf et al, 2014 & 1291.00 & 93 & 6.40 & 3.30 & 1.20 \\
Current study, 2021 & 3524.60 & 79 & 7.91 & 2.79 & 1.70 \\
\hline
\end{tabular}

CMSPMs, congenitally missing second permanent molars; IpH, prevalence of hypodontia; IpHSPM, prevalence of congenitally missing SPMs.

increasing prevalence set by the two previous reviews. Both previous reviews exhibited more missing lower SPMs, which are in accordance with the results presented here (Table IX).

\section{Conclusions}

The prevalence of CMSPMs is low compared with the overall prevalence of CM teeth. Significant differences in the prevalence of CMSPMs have been reported between continents, as well as between various geographical regions, in most cases with higher values for the mandible. Despite its rarity, early detection is important to enable practitioners to plan and start treatment at the best time for optimal results. Further studies on the association with other pathologies may allow early screening for diseases with later onset, such as colorectal or ovarian cancer, which can help improve patient outcomes. Future studies on CM teeth are required to ease comparisons and reduce risks for errors.

\section{Acknowledgements}

Not applicable.

\section{Funding}

No funding was received.

\section{Availability of data and materials}

Not applicable.

\section{Authors' contributions}

All authors substantially contributed to this review paper. Conception and design were the responsibility of ATF, $\mathrm{AD}$ and AV. RL, IAS, CF and AM performed the literature review. Analysis and interpretation of data was performed by ATF, AD, AV and AM. RL, IAS and CF drafted the initial manuscript and critically revised it for important intellectual content. Data authentication is not applicable. All authors have read and approved the final manuscript.

\section{Ethics approval and consent to participate}

Not applicable.

\section{Patient consent for publication}

Not applicable.

\section{Competing interests}

The authors declare that they have no competing interests. 


\section{References}

1. De Coster PJ, Marks LA, Martens LC and Huysseune A: Dental agenesis: Genetic and clinical perspectives. J Oral Pathol Med 38: 1-17, 2009.

2. Altug-Atac AT and Erdem D: Prevalence and distribution of dental anomalies in orthodontic patients. Am J Orthod Dentofacial Orthop 131: 510-514, 2007.

3. Goya HA, Tanaka S, Maeda T and Akimoto Y: An orthopantomographic study of hypodontia in permanent teeth of Japanese pediatric patients. J Oral Sci 50: 143-150, 2008.

4. Amini F, Rakhshan V and Babaei P: Prevalence and pattern of hypodontia in the permanent dentition of 3374 Iranian orthodontic patients. Dent Res J (Isfahan) 9: 245-250, 2012.

5. Meany S, Anweigi L, Ziada $\mathrm{H}$ and Allen F: The impact of hypodontia: A qualitative study on the experiences of patients. Eur J Orthod 34: 547-552, 2012.

6. Oshagh M, Salehi P, Pakshir H, Bazyar L and Rakhshan V: Associations between normative and self-perceived orthodontic treatment need in young-adult dental patients. Kor J Orthod 41: 440-446, 2011

7. Khosravanifard B, Ghanbari-Azarnir S, Rakhshan H, Sajjadi SH, Ehsan AM and Rakhshan V: Association between orthodontic treatment and need and masticatory performance. Orthodontics (Chic.) 13: e20-e18, 2012.

8. Chung CJ, Han JH and Kim KH: The pattern and prevalence of hypodontia in Koreans. Oral Dis 14: 620-625, 2008.

9. Aktan A, Kara I, Şener I, Bereket C, Ay S and Çiftçi M: Radiographic study of tooth agenesis in the Turkish population. Oral Radiol 26: 95-100, 2010.

10. Thesleff I: The genetic basis of tooth developement and dental defects. Am J Med Genet A 140: 2530-2535, 2006.

11. Al-Ani AH, Antoun JS, Thomson WM, Merriman TR and Farella M: Hypodontia: An update on its etiology, classification, and clinical management. Biomed Res Int 2017: 9378325, 2017.

12. Varela M, Arrieta P and Venturiera C: Non-syndromic concomitant hypodontia and supernumerary teeth in an orthodontic population. Eur J Orthod 31: 632-637, 2009.

13. Nieminen P, Arte S, Tanner D, Paulin L, Alaluusua S, Thesleff I and Pirinen S: Identification of a nonsense mutation in the PAX9 gene in molar oligodontia. Eur J Hum Gen 9: 743-746, 2001.

14. Vieira AR, Modesto A, Miera R, Barbos AR, Lidral AC and Murray JC: Interferon regulatory factor 6 (IRF6) and fibroblast growth factor receptor 1 (FGFR1) contribute to human tooth agenesis. Am J Med Genet A 143: 538-545, 2007.

15. Vieira AR, Meira R, Modesto A and Murray JC: MSX1, PAX9 and TGFA contribute to tooth agenesis in humans. J Dent Res 83: 723-727, 2004.

16. Frazier-Bowers SA, Guo DC, Cavender A, Xue L, Evans B King T, Milewicz D and D'Souza RN: A novel mutation in PAX9 causes molar oligodontia. J Dent Res 81: 129-133, 2002.

17. Fauzi NH, Ardini YD, Zainuddin Z and Lestari W: A review on non-syndromic tooth agenesis associated with PAX9 mutations. Jap Dent Sci Rev 54: 30-36, 2018.

18. Abdalla EM, Mostowska A, Jagodzinski PP, Dwidar K and Ismail SR: A novel WNT10A mutation causes non-syndromic hypodontia in an Egyptian family. Arch Oral Biol 59: 722-728, 2014.

19. Paranjyoti MV, Kumaraswamy KL, Begum LF, Manjunath K, Litha and Basher S: Tooth agenesis: A susceptible indicator for colorectal cancer? J Can Res Ther 14: 527-531, 2018.

20. Menezes R, Marazita ML, Goldstein McHenry T, Cooper ME, Bardi K, Brandon C, Letra A, Martin RA and Vieira AR: AXIS inhibition protein 2 , orofacial clefts and a family history of cancer. J Am Dent Assoc 140: 80-84, 2009.

21. Chalothorn LA, Beeman CS, Ebersole JL, Kluemper GT, Hicks EP, Kryscio RJ, DeSimone CP and Modesitt SC: Hypodontia as a risk marker for epithelial ovarian cancer: A case-controlled study. J Am Dent Assoc 139: 163-169, 2008.

22. Bonds J, Pollan-White S, Xiang L, Mues G and D'Souza R: Is there a link between ovarian cancer and tooth agenesis? Eur J Med Genet 57: 235-239, 2014.

23. Mattheeuws N, Dermaut L and Martens G: Has hypodontia increased in Caucasians during 20th century? A meta-analysis Eur J Orthod 26: 99-103, 2004.

24. Larmour CJ, Mossey PA, Thind BS, Forgie AH and Stirrups DR: Hypodontia-a retrospective of prevalence and etiology. Part I. Quintessence Int 36: 263-270, 2005.
25. Khalaf K, Miskelly J, Voge E and Macfarlanne T: Prevalence of hypodontia and associated factors: A systematic review and meta-analysis. J Orthod 41: 299-316, 2014

26. Mărgărit R, Andrei OC, Tănăsescu LA, Farcaşiu C, Bisoc A Dina MN, Burlibaşa M and Bodnar DC: Non-syndromic familial hypodontia: Rare case reports and literature review. Rom J Morphol Embryol 60: 1355-1360, 2019.

27. Schalk van der Weide Y, Prahl-Anderson B and Bosman F: Tooth formation in patients with oligodontia. Angle Orthod 63: 31-37, 1993.

28. www.aapd.org/globalassets/media/policies_guidelines/r_dentalgrowth.pdf.

29. Moher D, Liberati A, Tetzlaff J and Altman DG; PRISMA Group: Preferred reporting items for systematic reviews and meta-analyses: The PRISMA statement. PLoS Med 6: e1000097, 2009.

30. Ng'ang'a RN and Ng'ang'a PM: Hypodontia of permanent teeth in a Kenyan population. East Afr Med J 78: 200-203, 2001.

31. Albashaireh ZS and Khader YS: The prevalence and pattern of hypodontia of the permanent teeth and crown size and shape deformity affecting upper lateral incisors in a sample of Jordanian dental patients. Community Dent Health 23: 239-243, 2006.

32. Küchler EC, Risso PA, Costa Mde C, Modesto A and Vieira AR: Studies of dental anomalies in a large group of school children. Arch Oral Biol 53: 941-946, 2008.

33. Harris EF and Clark LL: Hypodontia: An epidemiologic study of American black and white people. Am J Orthod Dentofacial Orthop 134: 761-767, 2008

34. Aslam A, Naeem A and Arbab SS: Prevalence and distribution of hypodontia in Pakistani orthodontic population. Pak Oral Dent J 30: 406-411, 2010.

35. Ajami B, Shabzendedar M and Mehrjerdian M: Prevalence of hypodontia in nine- to fourteen-year-old children who attended the Mashhad school of dentistry. Indian J Dent Res 21: 549-551, 2010.

36. Bud S, Popsor S, Jurca AM, Suciu BC and Păcurar M: Statistical study on the prevalence of nonsyndromic hypodontia in youths. Act Medica Trans 2: 444-445, 2011.

37. Sheikhi M, Sadeghi MA and Ghorbanizadeh S: Prevalence of congenitally missing permanent teeth in Iran. Dent Res J (Isfahan) 9 (Suppl 1): S105-S111, 2012.

38. Cantekin K, Dane A, Miloglu O, Kazanci F, Bayrakdar S and Celikoglu M: Prevalence and intra-oral distribution of agenesis of permanent teeth among Eastern Turkish children. Eur J Paediatr Dent 13: 53-56, 2012.

39. Al-Jabaa AH and Aldrees AM: Prevalence of dental anomalies in Saudi orthodontic patients. J Contemp Dent Pract 14: 724-730, 2013.

40. Mani SA, Mohsin WS and John J: Prevalence and patterns of tooth agenesis among Malay children. Southeast Asian J Trop Med Public Health 45: 490-498, 2014.

41. Affan AA and Serour A: Prevalence of hypodontia in permanent dentition in a sample of Sudanese university students. Int Arab J Dent 5: 59-64, 2014

42. Hassan DA, Abuaffan AH and Hashim HA: Prevalence of hypodontia in a sample of Sudanese orthodontic patients. J Orthod Sci 3: 63-67, 2014

43. Kramerová L, Krejčí P, Kaplová E and Langová K: The incidence of tooth agenesis in pediatric patients in the Olomouc region (casual study). Ces Stomat Roč 114: 40-47, 2014.

44. Shokri A, Poorolajal J, Khajeh S, Faramarzi F and Kahnamoui HM: Prevalence of dental anomalies among 7- to 35-year-old people in Hamadan, Iran in 2012-2013 as observed using panoramic radiographs. Imag Sci Dent 44: 7-13, 2014

45. Majeed M, Ahmed I, Uzair M and Atif M: Prevalence of missing, impacted and supernumerary teeth in patients under orthodontic treatment in a teaching hospital of Karachi, Pakistan. Int J Dent Health Sci 1: 39-46, 2014.

46. Al Jawad AFH, Al Yafei H, Al Sheeb M, Al Emadi B and Al Hashimi N: Hypodontia prevalence and distribution pattern in a group of Qatari orthodontic and pediatric patients: A retrospective study. Eur J Dent 9: 267-221, 2015.

47. Zhang J, Liu HC, Lyu X, Shen GH, Deng XX, Li WR, Zhang XX and Feng HL: Prevalence of tooth agenesis in adolescent Chinese populations with or without orthodontics. Chin J Dent Res 18: 59-65, 2015.

48. Hashim HA and Al-Said S: The prevalence and distribution of hypodontia in a sample of Qatari patients. J Orthod Sci 5: 1-6, 2016. 
49. Gokkaya B and Kargul B: Prevalence and pattern of non-syndromic hypodontia in a group of Turkish children. Acta Stomatol Croat 50: 58-64, 2016.

50. Sajjad A, Sajjad SA, Husain N and Al-Enezi AM: A retrospective cross-sectional study on the prevalence of hypodontia in a target population of Al-Jouf Province, Saudi Arabia. Contemp Clin Dent 7: 500-505, 2016.

51. Al-Abdallah M, Hadidi A, Hammad M, Al-Ahmad H and Saleh R: Prevalence and distribution of dental anomalies: A comparison between maxillary and mandibular tooth agenesis. Am J Orthod Dentofacial Orthop 148: 793-798, 2015.

52. Ameen RM, Abduljabbar OF and Saleh MH: Prevalence of hypodontia of permanent dentition in a sample of orthodontic patients In Erbil City: An orthopantomograph study. Zanco J Med Sci 21: 1751-1756, 2017.

53. Sola RA, Sola PA, Pérez JC, Sánchez IN and Renovales ID Prevalence of hypodontia in a sample of Spanish dental patients Acta Stomatol Croat 52: 18-23, 2018.

54. Reshitaj A, Krasniqi D, Reshitaj K and Anic Milosevic S Hypodontia, gender-based differences and its correlation with other dental clinical features in Kosovar adolescents. Acta Stomatol Croat 53: 347-353, 2019.

55. Georgescu DE, Georgescu EM and Luca R: Prevalence and distribution of dental developmental anomalies-orthopantomographic study. Ro J Stomatol 65: 389-394, 2019.

56. Musaed ZAN, Al-Haraz G and Al-Deen HS: Prevalence of congenitally missing permanent teeth in a group of Yemen population: A radiographic study. Saudi J Oral Dent Res 4: 704-715, 2019.

57. Farcașiu C, Farcașiu AT, Moşneanu AM and Andrei OC: Retrospective statistical study regarding dental number anomalies in permanent dentition on two groups of children in Bucharest. DentalTarget 15: 65-70, 2020.

58. Fekonja A: Hypodontia in orthodontically treated children. Eur J Ortho 27: 457-460, 2005

59. Endo T, Ozoe R, Kubota M, Akiyama M and Shimooka S: A survey of hypodontia in Japanese orthodontic patients. Am J Orthod Dentofacial Orthop 129: 29-35, 2006.

60. Sisman Y, Uysal T and Gelgor IE: Hypodontia. Does the prevalence and distribution pattern differ in orthodontic patients? Eur J Dent 1: 167-173, 2007

61. Maatouk F, Baaziz A, Ghnima S, Masmoudi F and Ghedira H: Survey on hypodontia in Sayada, Tunisia. Quintessence Int 39: e115-e120, 2008

62. Abu Sakra K and Alqaqaa S: Prevalence of hypodontia and oligodontia in a sample of orthodontic and pediatric dentistry patients at Prince Hashem bin Alhussein military hospital. J R Med Serv 15: 40-44, 2008.

63. Rølling S and Poulsen S: Agenesis of permanent teeth in 8138 Danish schoolchildren: Prevalence and intra-oral distribution according to gender. Int J Paediatr Dent 19: 172-175, 2009.

64. Peker I, Kaya E and Darendeliler-Yaman S: Clinic and radiographical evaluation of non-syndromic hypodontia and hyperdontia in permanent dentition. Med Oral Patol Oral Cir Bucal 14: e393-e397, 2009

65. Gomes RR, da Fonseca JA, Paula LM, Faber J and Acevedo AC: Prevalence of hypodontia in orthodontic patients in Brasilia, Brazil. Eur J Orthod 32: 302-306, 2010.

66. Topkara A and Sari Z: Prevalence and distribution of hypodontia in a Turkish orthodontic patient population: Results from a large academic cohort. Eur J Paed Dent 12: 123-127, 2011.

67. Vahid-Dastjerdi E, Borzabadi-Farahani A, Mahdian M and Amini N: Non-syndromic hypodontia in an Iranian orthodontic population. J Oral Sci 52: 455-461, 2010.

68. Tallón-Walton V, Nieminen P, Arte S, Carvalho-Lobato P, Ustrell-Torrent JM and Manzanares-Céspedes MC: An epidemiological study of dental agenesis in a primary health area in Spain: Estimated prevalence and associated factors. Med Oral Patol Oral Cir Bucal 15: e569-e574, 2010.

69. Carvalho S, Mesquitab P and Afonso A: Prevalence of dental anomalies of number in one Portuguese population. A radiographic study. Rev Port Estomatol Med Dent Cir Maxilofac 52: 7-12, 2011.

70. Kim YH: Investigation of hypodontia as clinically related dental anomaly: Prevalence and characteristics. ISRN Dent 2001: 246135,2011

71. Kazanci F, Celikoglu M, Miloglu O, Ceylan I and Kamak H: Frequency and distribution of developmental anomalies in the permanent teeth of a Turkish orthodontic patient population. J Dent Sci 6: 82-89, 2011
72. Ide M, Kuriyama C, Takeuchi C, Takamizawa Y, Takamizawa S, Namba T, Tomohiro T and Asada Y: The prevalence of congenitally missing permanent teeth in Japanese children: A survey conducted in south-west area of Kanto district. Ped Dent J 21: 24-30, 2011.

73. Coelho AS, Macho VM, Andrade DJ, Augusto AP and Areias CM: Prevalence and distribution of tooth agenesis in a pediatric population: A radiographic study. Rev Gaúch Odontol 60: 503-508, 2012.

74. Medina AC: Radiographic study of prevalence and distribution of hypodontia in a pediatric orthodontic population in Venezuela. Ped Dent 34: 113-116, 2012.

75. de Freitas DQ, Tsumurai RY and Machado Filho DN: Prevalence of dental anomalies of number, size, shape and structure. Rev Gaúcha Odontol 60: 437-441, 2012.

76. Uzuner D, Celik MM, Toy E and Turkdonmez CO: Assessment of hypodontia in the Turkish patients referring to the orthodontic clinic: A retrospective study. Eur J Dent 7 (Suppl 1): S9-S14, 2013.

77. González-Allo A, Campoy CM, Moreira J, Ustrell J and Pinho T: Tooth agenesis in a Portuguese population. Int Orthod 10: 198-210, 2012

78. Hyunsoon J, Yeonmi Y, Byeongju B and Jaegon K: Prevalence and distribution of congenitally missing teeth in patients visiting the department of pediatric dentistry of Chonbuk national university hospital. J Korean Acad Pediatr Dent 40: 274-282, 2013.

79. Kerekes-Máthé B, Mártha K and Székely M: Prevalence and characteristics of tooth agenesis in permanent dentition of subjects from Tîrgu Mureș. Acta Medica Maris 59: 187-190, 2013.

80. Al-Amiri A, Tabbaa S, Preston CB and Al-Jewair T: The prevalence of dental anomalies in orthodontic patients at the State University of New York at Buffalo. J Contemp Dent Pract 14: 518-523, 2013.

81. Hedayati Z and Dashlibrun YN: The prevalence and distribution pattern of hypodontia among orthodontic patients in Southern Iran. Eur J Dent 7 (Suppl 1): S78-S82, 2013.

82. Bozga A, Stanciu RP and Mănuc D: A study of prevalence and distribution of tooth agenesis. J Med Life 7: 551-554, 2014.

83. Herrera-Atoche JR, Diaz-Morales SM, Colomé-Ruiz GE, Escoffié-Ramírez M and Orellana MF: The prevalence of dental anomalies in a Mexican population. Denstry 3000 2: 1-5, 2014.

84. Pop Acev D and Gjorgova J. Prevalence of hypodontia in the permanent dentition of Macedonian population. Balk J Dent Med 18: 93-98, 2014.

85. Abu-Hussein M, Watted N, Watted A, Abu-Hussein Y, Yehia M, Awadi $\mathrm{O}$ and Azzaldeen A: Prevalence of tooth agenesis in orthodontic patients at Arab population in Israel. Int J Public Health Res 3: 77-82, 2015.

86. Dang HQ, Constantine S and Anderson PJ: The prevalence of dental anomalies in an Australian population. Aust Dent J 62: 161-164, 2017.

87. Hagiwara Y, Uehara T, Narita T, Tsutsumi H, Nakabayashi S and Araki M: Prevalence and distribution of anomalies of permanent dentition in 9584 Japanese high school students. Odontology 104: 380-389, 2016

88. Badrov J, Gaspar G, Tadin A, Galic T, Govorko DK, Gavic L, Badrov R and Galic I: Prevalence and characteristics of congenitally missing permanent teeth among orthodontic patients in Southern Croatia. Acta Stomatol Croat 51: 290-299, 2017.

89. Gracco AL, Zanatta S, Forin Valvecchi F, Bignotti D, Perri A and Baciliero F: Prevalence of dental agenesis in a sample of Italian orthodontic patients: An epidemiological study. Prog Orthod 18: 33, 2017

90. Laganà G, Venza N, Borzabadi-Farahani A, Fabi F, Danesi C and Cozza P: Dental anomalies: Prevalence and associations between them in a large sample of non-orthodontic subjects, a cross-sectional study. BMC Oral Health 17: 62, 2017.

91. Souza-Silva BN, Vieira WA, Bernardino ÍM, Batista MJ, Vieira Bittencourte MA and Paranhos LR: Non-syndromic tooth agenesis patterns and their association with other dental anomalies: A retrospective study. Arch Oral Biol 96: 26-32, 2018.

92. Ifesanya JU, Temisanren OT and Jaiyeoba OO: A radiographic assessment of the prevalence and pattern of dental agenesis in a Nigerian population. African J Oral Health 8: 10-15, 2018.

93. Altan H, Cosgun A, Akkoc S and Erağca R: Are environmental factors effective in congenitally missing teeth? A multicenter study. Pesq Bras Odont Clin Integ 19: e4879, 2019. 
94. Fauzia NH, Lestarib W, Kharuddina AF and Ardini YD: Prevalence, pattern and distribution of non-syndromic tooth agenesis in permanent dentition among Malaysian population. Mat Today Proc 16: 2204-2209, 2019.

95. Alhaddad AS, Al-Hur AA, Alyessary AA and Abbas GA Prevalence of congenital missing permanent teeth in a sample of Iraqi patients attending dental clinic of Kerbala university: A retrospective study. Biochem Cell Arch 19: 3265-3272, 2019.

96. Kielan-Grabowska Z, Kawala B and Antoszewska-Smith J: Hypodontia-not only an orthodontic problem. Dent Med Probl 56: 373-377, 2019.

97. Wiener RC and Waters C: Hypodontia, oligodontia and anodontia in West Virginia Appalachia. J Dent Hyg 93: 34-40, 2019.

98. Chan GXL, Tan ELY, Chew MT, Wong HC, Foong KWC and Yow M: Secondary dentition characteristics in an ethnic Chinese orthodontic population: A retrospective cross-sectional study. J Investig Clin Dent 10: e12421, 2019.

99. De Stefani A, Bruno G, Conte E, Frezza A, Balasso P and Gracco A: Prevalence and patterns of tooth agenesis in Angle class II division 2 malocclusion in Italy: A case-control study. Int Orthod 17: 538-543, 2019.

100. Gupta SP and Rauniyar S: Prevalence and distribution of dental anomalies among orthodontic patients of Kathmandu, Nepal. Ortho J Nepal 9: 23-28, 2020.

101. Çakir A and Yildirim S: Prevalence of congenital missing permanent teeth in children group aged 5-14 years living in the Konya region: Retrospective study. J Int Dent Sci 2: 12-17, 2020.
102. Rakhshan V: Meta-analysis of observational studies on the most commonly missing permanent dentition (excluding the third molars) in non-syndromic dental patients or randomly-selected subjects, and the factors affecting the observed rates. J Clin Pediatr Dent 39: 198-207, 2015.

103. Brook AH: A unifying aetiological explanation for anomalies of human tooth number and size. Arch Oral Biol 29: 373-378, 1984.

104. Polder BJ, Van't Hof MA, Van der Linden FP and KuijpersJagtman AM: A meta-analysis of the prevalence of dental agenesis of permanent teeth. Community Dent Oral Epidemiol 32: 217-226, 2004

105. Bondemark L and Tsiopa J: Prevalence of ectopic eruption, impaction, retention and agenesis of the permanent second molar. Angle Orthod 77: 773-778, 2007.

106. Rakhshan V and Rakhshan R: Meta-analysis and systematic review of the number of non-syndromic congenitally missing permanent teeth per affected individual and its influencing factors. Eur J Orth 38: 170-177, 2016.

c) (i) $\Theta$ This work is licensed under a Creative Common International (CC BY-NC-ND 4.0) License. 